\title{
Intensive exercise and a patient in acute phase of polymyositis
}

\begin{abstract}
Background and objective: Polymyositis (PM) is an idiopathic inflammatory myopathy manifested by proximal limb muscles weakness, elevated creatinin kinase, electromyography changes, and muscle inflammation in biopsy. We report an instance of intensive rehabilitation therapy in a patient with clinically active polymyositis. Case report: A 19-year-old female patient, diagnosed with 'electromyography and biopsy proven' polymyositis for 5 years, suffered from worsening limbs weakness and dysphagia. In her history, she had upper and lower limbs weakness accompanied by dysphagia which was further complicated by right bronchial aspiration 9 months ago. A four-week trial of intensive training and exercise rehabilitation, concurrently accompanied by medications was prescribed for this patient. At the end of therapy she achieved significant improvement in muscle strength, activities of daily living, and ambulation without any disease exacerbation. Conclusion: We concluded that short-term intensive training and exercise may lead to improvements in patients with PM, without causing a progress in the disease. Due to the rarity of PM and difficulty of conducting well-controlled studies to examine the risks and benefits of exercise in these patients, further research is necessary to investigate benefits of exercise training in active phase of disease.
\end{abstract}

Keyword: Creatinin kinase; Electromyography; Idiopathic inflammatory myopathy; Polymyositis; Rehabilitation 